\title{
Pseudo-concave integrals
}

\author{
Radko Mesiar ${ }^{1,2},{\mathrm{Jun} \mathrm{Li}^{3} \text {, and Endre Pap }}^{4,5}$
}

\begin{abstract}
The notion of Lehrer-concave integral is generalized taking instead of the usual arithmetical operations of addition and multiplication of reals more general real operations called pseudo-addition and pseudomultiplication.
\end{abstract}

\section{Introduction}

The integration theory makes a fundament for the classical measure theory, see [16]. The Riemann and the Lebesgue integrals are related to the additive measure (more precisely countably additive measure), which makes the base also for the probability theory. The first integral based on non-additive measures (first of all on monotone measure or capacity) was the Choquet integral [2], see [3, 15], which also covers the classical Lebesgue integral. On the other side, Sugeno [18] has introduced an integral based also on non-additive measures, but with respect to join (maximum) and meet (minimum) operations instead of the usual addition and product. In these approaches it was important the horizontal representation by means of the level sets of a fuzzy subset of a universe $X$, where the level set for level $t$ consists of all points of $X$ with degree of membership greater than or equal to $t$. The Choquet integral is based also on the horizontal approach. Further generalizations of integrals related the horizontal approach is given under universal integral [7].

\footnotetext{
${ }^{1}$ Department of Mathematics and Descriptive Geometry, Faculty of Civil Engineering, Slovak University of Technology, Radlinského 11, 81368 Bratislava, Slovakia mesiar@math.sk 2 UTIA CAS, P.O.Box 18, 18208 Prague, Czech Republic

${ }^{3}$ School of Science, Communication University of China, Beijing, 100024, People's Republic of China lijun@cuc.edu.cn

4 Department of Mathematics and Informatics, University of Novi Sad, Trg Dositeja Obradovića 4, 21000 Novi Sad, Serbia pape@eunet.rs

5 Óbuda University, Becsi út 96/B, H-1034 Budapest, Hungary
} 
The horizontal approach based on level sets has as a consequence that chains of subsets of $X$ play the important role. Lehrer $[8,9]$ has introduced concave integral which is not based on horizontal approach, but considering all possible partitions. His integral coincides with the Choquet integral only when the monotone measure (capacity) is convex (supermodular).

In this paper we investigate the generalization of the Lehrer integral taking more general real operations than classical plus and product. For this purpose we shall use pseudo-addition $\oplus$ and pseudo-multiplication $\odot$, see $[1,15,19]$.

\section{Definition of Lehrer integral}

Let $\Omega$ be a non-empty set, $\mathcal{A}$ a $\sigma$-algebra of subsets of $\Omega$ and $v: \mathcal{A} \rightarrow[0, \infty[$ a monotone set function with $v(\varnothing)=0$. Two well-known nonadditive integrals are Choquet and Sugeno integrals, see [15]. The Choquet integral [2] of a measurable nonnegative function $f$ is given by

$$
\begin{gathered}
\mathcal{C}_{v}(f)=\int_{0}^{\infty} v(f \geqslant t) d t \\
=\sup \left\{\sum_{i \in I} a_{i} v\left(A_{i}\right) \mid \sum_{i=1}^{N} a_{i} \mathbf{1}_{A_{i}} \leqslant f,\left(A_{i}\right)_{i=1}^{N} \subset \mathcal{A} \text { decreasing, } a_{i} \geqslant 0, N \in \mathbb{N}\right\},
\end{gathered}
$$

where $A_{i+1} \subseteq A_{i}$ for every $i=1,2, \ldots, N-1$.

The Choquet integral has the property of the reconstruction of the measure, i.e., for every $A \in \mathcal{A}$ we have

$$
\int_{0}^{\infty} \mathbf{1}_{A} d v=v(A)
$$

The Choquet integral for finite case, step functions, is given on $X=$ $\{1, \ldots, n\}$ and for $\left(a_{1}, a_{2}, \ldots, a_{n}\right) \in\left[0, \infty\left[^{n}\right.\right.$ by

$$
\mathcal{C}_{v}\left(a_{1}, a_{2}, \ldots, a_{n}\right)=\sum_{i=1}^{n}\left(a_{(i)}-a_{(i-1)}\right) v\left(A_{(i)}\right)
$$

with a permutation $\sigma$ on $\{1, \ldots, n\}$ such that $a_{(1)} \leqslant a_{(2)} \leqslant \cdots \leqslant a_{(n)}$, with the convention $x_{(0)}=0$, and $A_{(i)}=\{(i), \ldots,(n)\}$. The Choquet integral can be given by an equivalent formula

$$
\mathcal{C}_{v}\left(a_{1}, a_{2}, \ldots, a_{n}\right)=\sum_{i=1}^{n} a_{(i)}\left(v\left(A_{(i)}\right)-v\left(A_{(i+1)}\right)\right),
$$

with $A_{(n+1)}=\varnothing$. The Sugeno integral [18] is given on $X=\{1, \ldots, n\}$ and for $\left(a_{1}, a_{2}, \ldots, a_{n}\right) \in\left[0, \infty\left[^{n}\right.\right.$ by 


$$
\mathcal{S}_{v}\left(a_{1}, a_{2}, \ldots, a_{n}\right)=\bigvee_{i=1}^{n}\left(a_{(i)} \wedge v\left(A_{(i)}\right)\right) .
$$

One integral is also introduced, namely, the Shilkret integral [17] given by

$$
\mathcal{K}_{v}(f)=\sup \{t \cdot v(\{f \geqslant t\}) \mid t \in[0, \infty]\} .
$$

A set function $v$ on $2^{N}$ is supermodular, i.e., 2-monotone or convex, if it satisfies the following inequality for all $A, B \in 2^{N}$ :

$$
v(A \cup B)+v(A \cap B) \geqslant v(A)+v(B) .
$$

The functional $f \mapsto \mathcal{C}_{v}(f)$ is concave if and only if $v$ is supermodular, see [10].

Lehrer concave integral is given in the following definition, [9].

Definition 1. Concave integral of a measurable function $f: \Omega \rightarrow[0, \infty[$ is given by

$$
(L) \int f d v=\sup \left\{\sum_{i \in I} a_{i} v\left(A_{i}\right) \mid \sum_{i \in I} a_{i} \mathbf{1}_{A_{i}} \leqslant f, I \text { is finite, } a_{i} \geqslant 0\right\},
$$

where $A_{i}, i \in I$, are measurable.

\section{Pseudo-operations}

We have seen that the Lehrer-concave integral as also the Choquet integral are strongly related to the usual operations of addition + and multiplication $\cdot$ on the interval $[0, \infty]$. Similarly, the Sugeno integral depends on the operations $\vee$ and $\wedge$ on the interval $[0,1]$.

There were considered generalizations of the previously mentioned operations $[1,6,19]$. In order to find a common framework for both Choquet and Sugeno integrals, we have to deal with a general pseudo-addition $\oplus$ and a general pseudo-multiplication $\odot$ which must be fitting to each other. In general, $\oplus$ is supposed to be a continuous generalized triangular conorm (see $[1,5,15])$.

Definition 2. A binary operation $\oplus:[0, \infty]^{2} \rightarrow[0, \infty]$ is called a pseudoaddition on $[0, \infty]$ if the following properties are satisfied:

(PA 1) $a \oplus b=b \oplus a$ (commutativity)

(PA 2) $a \leq a^{\prime}, b \leq b^{\prime} \Rightarrow a \oplus b \leq a^{\prime} \oplus b^{\prime}$ (monotonicity)

(PA 3) $(a \oplus b) \oplus c=a \oplus(b \oplus c)$ (associativity) 
(PA 4) $a \oplus 0=0 \oplus a=a$ (neutral element)

(PA 5) $a_{n} \rightarrow a, b_{n} \rightarrow b \Rightarrow a_{n} \oplus b_{n} \rightarrow a \oplus b$ (continuity).

Remark 1. The structure of the operation $\oplus$ is described in details as an $I$-semigroup, see for more details in [5].

By (PA 5), the continuity of the operation $\oplus$, the set of $\oplus$-idempotent elements $C_{\oplus}=\{a \in[0, \infty], a \oplus a=a\}$ is closed and non-empty since $0, \infty \in$ $C_{\oplus}$. Two extreme cases are possible: $C_{\oplus}=\{0, \infty\}$ and $C_{\oplus}=[0, \infty]$. In the first case the pseudo-addition $\oplus$ is isomorph with the usual addition on $[0, \infty]$ or isomorph with the truncated addition on $[0,1]$. All other cases are covered by ordinal sums of the first case, $[5,19]$.

For the integration procedure we need another binary operation $\odot$, which is called pseudo-multiplication. The expected properties of the integral determine the next minimal properties of $\odot$ which we have to require, see $[5,19]$.

Definition 3. Let $\oplus$ be a given pseudo-addition on $[0, \infty]$. A binary operation $\odot:[0, \infty] \times[0, \infty]$ is called a $\oplus$-fitting pseudo-multiplication if the following properties are satisfied:

(PM 1) $a \odot 0=0 \odot b=0$ (zero element)

(PM 2) $a \leq a^{\prime}, b \leq b^{\prime} \Rightarrow a \odot b \leq a^{\prime} \odot b^{\prime}$ (monotonicity)

(PM 3) $(a \oplus b) \odot c=a \odot c \oplus b \odot c$ (left distributivity)

(PM 4) $\left(\sup _{n} a_{n}\right) \odot\left(\sup _{m} b_{m}\right)=\sup _{n, m} a_{n} \odot b_{m}$ (left continuity).

\section{Pseudo-concave integral}

For any $a \in \overline{\mathbb{R}}^{+}$and any $A \in \mathcal{A}$, the function $b(a, A)$ defined by:

$$
b(a, A)(x)=\left\{\begin{array}{l}
a \text { if } x \in A \\
0 \text { if } x \notin A .
\end{array}\right.
$$

is called basic (simple) function.

We can introduce a generalization of Lehrer's integral with respect to pseudo-addition $\oplus$ and pseudo-multiplication $\odot$.

Definition 4. Let $\oplus:[0, \infty]^{2} \rightarrow[0, \infty]$ be a pseudo-addition and $\odot$ : $[0, \infty]^{2} \rightarrow[0, \infty]$. Pseudo-concave integral of a measurable function $f: \Omega \rightarrow$ $[0, \infty[$ is given by

$(L) \int^{\oplus, \odot} f d v=\sup \left\{\bigoplus_{i \in I} a_{i} \odot v\left(A_{i}\right) \mid \bigoplus_{i \in I} b\left(a_{i}, A_{i}\right) \leqslant f, I\right.$ is finite,$\left.a_{i} \geqslant 0\right\}$, 
where $A_{i}, i \in I$, are measurable.

Example 1. (i) Of course that for $\oplus=+$ and $\odot=$. we obtain the Lehrer's integral from Definition 1.

(ii) In a special case, when $\oplus=\vee$, the corresponding pseudo-multiplication have to be non-decreasing, and then we have

$$
\left.(L) \int^{\vee, \odot} f d v=\bigvee_{a \in[0, \infty]} a \odot v(f \geqslant a)\right) .
$$

This case cover Sugeno $\mathcal{S}_{v}$ and Shilkret $\mathcal{K}_{v}$ integrals, taking for the pseudomultiplication $\odot$ minimum $\wedge$ and product $\cdot$, respectively.

(iii) In another special case, when $\oplus$ is strict (strictly monotone), then there exists an increasing bijection $g:[0, \infty] \rightarrow[0, \infty]$ such that

$$
a \oplus b=g^{-1}(g(a)+g(b)),
$$

see [19]. The only left distributive pseudo-multiplication $\odot$ is given by

$$
a \odot b=g^{-1}(g(a) h(b)),
$$

where $h:[0, \infty] \rightarrow[0, \infty]$ is a non-decreasing function, see $[13,14]$. Then we have

$$
\begin{aligned}
&(L) \int^{\oplus, \odot} f d v= \sup \left\{g ^ { - 1 } \left(\sum_{i \in I} a_{i} h\left(v\left(A_{i}\right)\right) \mid g^{-1}\left(\sum_{i \in I} b\left(g\left(a_{i}\right), A_{i}\right)\right) \leqslant f\right.\right. \\
&\left.I \text { is finite }, a_{i} \geqslant 0\right\} \\
&=g^{-1}\left((L) \int g \circ f d h \circ v\right) .
\end{aligned}
$$

For $h=g$ we obtain convex $g$-integral, for $g$-like integral see [11].

Depending on additional properties of pseudo-multiplication $\odot$ we can obtain useful properties of pseudo-concave integral. For example, if $\odot$ is associative we obtain the positive homogeneity of pseudo-concave integral. Generally, we have for two measurable functions $f_{1}$ and $f_{2}$

$$
(L) \int^{\oplus, \odot}\left(f_{1} \oplus f_{2}\right) d v \geqslant(L) \int^{\oplus, \odot} f_{1} d v \oplus(L) \int^{\oplus, \odot} f_{2} d v .
$$




\section{Concluding remarks}

Our proposal of pseudo-concave integrals can be seen as a starting point for a deeper investigation of properties of this interesting functional, promissing fruitful applications in the area of multicriteria decision making and related areas. Note that a related concept generalizing Lebesgue integral was recently proposed and discussed in [21], and it would be interesting to see the connections with the pseudo-concave integral. Another line for deeper study of pseudo-concave integrals can done for integral inequalities and relations with some classical integrals, in the spirit of our recent work [12].

Acknowledgements The first author was supported by the grants APVV-0073-10 and P402/11/0378. The second author was supported by NSFC Grant No.70771010. The third author was supported by the national grants MNTRS (Serbia, Project 174009), and "Mathematical models of intelligent systems and their applications" by Provincial Secretariat for Science and Technological Development of Vojvodina.

\section{References}

1. Benvenuti P, Mesiar R, Vivona D (2002) Monotone set functions-based integrals. In: Pap E. (ed.) Handbook of Measure Theory, Vol. II. Elsevier Science, 1329-1379

2. Choquet G (1954) Theory of capacities. Ann. Inst. Fourier (Grenoble) 5:131295

3. Denneberg D (1994) Non-Additive Measure and Integral. Kluwer Academic Publishers, Dordrecht

4. Klement E.P, Mesiar R, Pap E (1996) On the relationship of associative compensatory operators to triangular norms and conorms. Internat. J. Uncertainty, Fuzziness and Knowledge-Based Systems 4, 25-36

5. Klement E.P, Mesiar R, Pap E (2000a) Triangular Norms. Kluwer Academic Publishers, Dordrecht

6. Klement E.P, Mesiar R, Pap E (2000b) Integration with respect to decomposable measures, based on a conditionally distributive semiring on the unit interval. Internat. J. Uncertain. Fuzziness Knowledge-Based Systems 8:701-717

7. Klement E. P, Mesiar R, Pap E (2010) A universal integral as common frame for Choquet and Sugeno integral. IEEE Transactions on Fuzzy Systems 18:178-187

8. Lehrer E (2009) A new integral for capacities. Econ. Theory 39:157-176

9. Lehrer E, Teper R (2008) The concave integral over large spaces. Fuzzy Sets and Systems 159:2130-2144

10. Lovász L (1983) Submodular functions and convexity. In A. Bachem, M. Grötschel, and B. Korte, editors, Mathematical programming. The state of the art, Springer Verlag, 235-257

11. Mesiar R (1995) Choquet-like integral. J. Math. Anal. Appl. 194:477-488

12. Mesiar R, Li J, Pap E (2010) The Choquet integral as Lebesgue integral. Kybernetika 46: 931-934

13. Mesiar R, Rybárik J (1995) PAN-operations. Fuzzy Sets and Systems 74:365-369

14. Murofushi T, Sugeno M. (1991) Fuzzy t-conorm integral with respect to fuzzy measures: Generalization of Sugeno integral and Choquet integral. Fuzzy Sets and Systems 42:57-71

15. Pap E (1995) Null-Additive Set Functions. Kluwer, Dordrecht 
16. Pap E (Ed.) (2002) Handbook of Measure Theory. Amsterdam, The Netherlands: Elsevier

17. Shilkret, N (1971) Maxitive measure and integration. Indag. Math. 33:109-116

18. Sugeno, M (1974) Theory of fuzzy integrals and its applications. Ph. D. Thesis, Tokyo Institute of Technology

19. Sugeno M, Murofushi T. (1987) Pseudo-additive measures and integrals. J. Math. Anal. Appl. 122: 197-222

20. Wang Z, Klir G. J (2009) Generalized Measure Theory. Springer, Boston

21. Zhang Q, Mesiar R, Li J, Struk P (2011) Generalized Lebesgue integral. Int. J. Approximate Reasoning 52:427-443. 